\title{
Pour une réforme en profondeur de l'Agence Nationale de la Recherche
}

Dans le contexte d'un enseignement supérieur et d'une recherche en constante évolution, quel est l'équilibre souhaitable entre les financements des différents opérateurs ? La priorité doit être donnée à une augmentation significative des crédits de base des laboratoires. Faut-il conserver l'Agence Nationale de la Recherche ? On peut proposer une agence dont les missions et le fonctionnement seraient repensés pour tenir compte des critiques formulées par ses utilisateurs.

La répartition des crédits pour la recherche et l'innovation est, en France comme dans tous les pays, un enjeu critique. Il y a encore dix ans, ces missions étaient accomplies de manière très professionnelle par le CNRS et les autres organismes de recherche, ainsi que par le ministère de la Recherche, notamment avec les Actions Concertées Incitatives. Le paysage a depuis considérablement changé. Les établissements d'enseignement supérieur sont devenus autonomes, et la création de l'Agence Nationale de la Recherche (ANR) en 2006 a renforcé la culture de la recherche sur projet. Simultanément les moyens des grands organismes comme le CNRS se sont rétrécis, asséchant progressivement les financements de base fournis aux laboratoires.

\section{Faut-il conserver une agence nationale de recherche?}

Des moyens significatifs et bien appréciés ont certes été injectés dans la recherche depuis quelques années; cependant, la nouvelle organisation du financement a certains inconvénients. Le rôle du CNRS et des organismes s'est affaibli, en se concentrant sur l'attribution et la gestion des postes et le maintien des grandes infrastructures de recherche. La vie collective d'un laboratoire est de plus en plus difficile à assurer quand les crédits récurrents deviennent quasiment nuls. Il faut donc affirmer avant tout que les organismes, au côté des universités, doivent retrouver une capacité à financer les laboratoires, grâce à une augmentation substantielle des crédits de base. Les directeurs des laboratoires pourront ainsi retrouver les moyens de financer les infrastructures, et établir une politique scientifique cohérente avec l'ensemble de leurs équipes. En outre, les universités doivent pouvoir financer des initiatives de recherche propres.

Peut-on pour autant se passer en France d'une agence nationale de recherche, comme certains le préconisent ? Faut-il reconcentrer tous les moyens sur le CNRS et les organismes? Outre qu'il n'est pas certain qu'on y retrouvera l'intégralité du budget de l'ANR, on doit aujourd'hui tenir compte de la multiplicité des opérateurs de la recherche, ainsi que de l'autonomie des universités. Comme pour tous les grands pays de recherche dans le monde, il semble nécessaire qu'une part importante des crédits de recherche soit répartie sur la base d'une évaluation nationale et interdisciplinaire, de manière à assurer une qualité optimale et éviter les conflits d'intérêt.

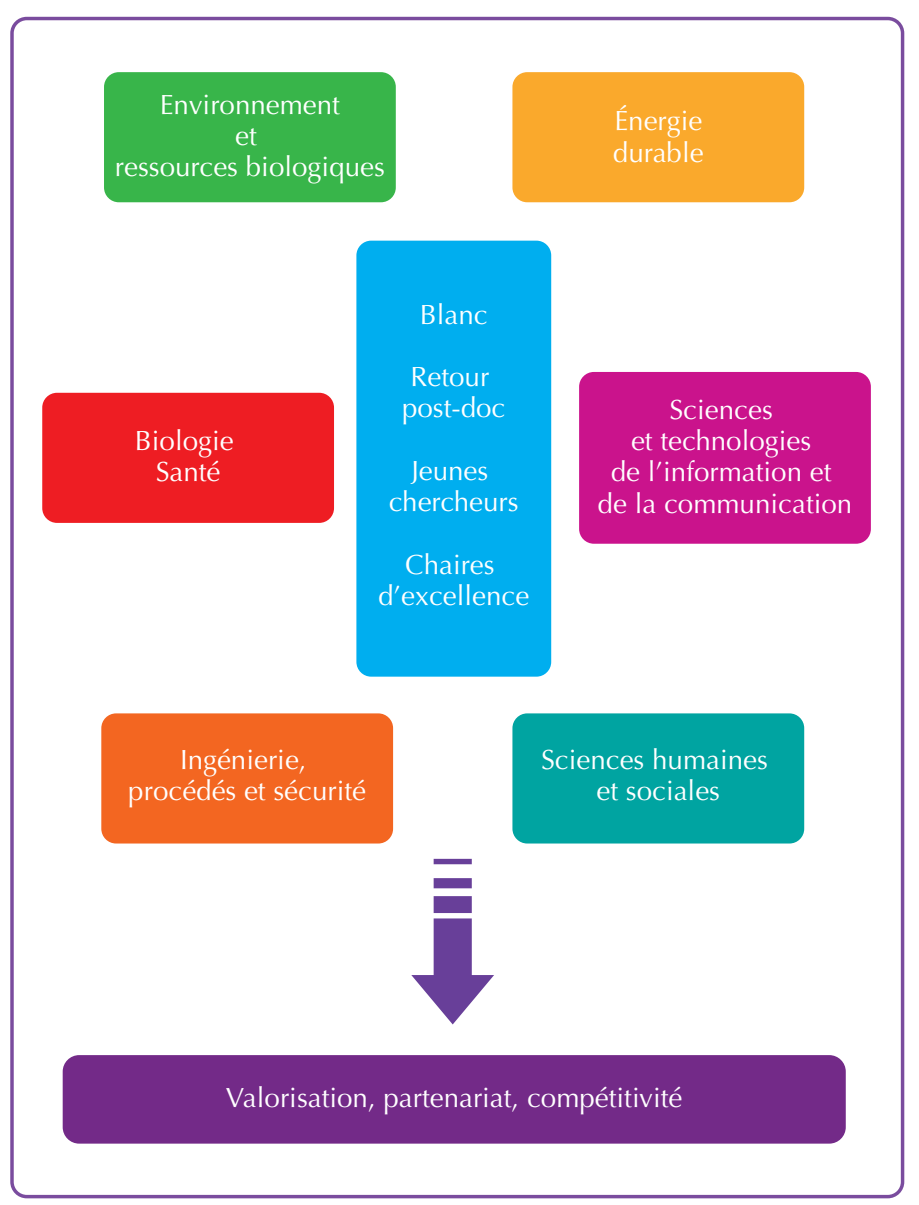

Les programmes de l'Agence Nationale de la Recherche pour l'année 2012. (Source : www.agence-nationale-ececherche.fr).

Sans repartir à zéro, nous pensons donc qu'il faut maintenir une agence de la recherche en France, en soutien à la recherche publique. Dans un contexte budgétaire contraint, surtout si les organismes et les universités voient, comme il est souhaitable, leurs moyens augmenter, il convient de mener une véritable réflexion sur la stratégie de financement d'une agence, en corrigeant ses défauts actuels et en cohérence avec les autres acteurs de la recherche. 


\section{Libre opinion}

\section{L'ANR vue par beaucoup de ses utilisateurs}

\section{Le taux de sélection est beaucoup trop faible.}

Le taux de sélection est maintenant au mieux de $20 \%$ pour les programmes «blancs » et " jeunes » et dans la plupart des comités thématiques, alors qu'il était de près de $27 \%$ en 2006. Ces taux de succès trop faibles vont avec l'augmentation du nombre de dossiers, l'un entrainant l'autre dans un processus divergent. La raison en est, d'une part la baisse depuis deux ans des crédits de l'ANR destinés aux projets de recherche, d'autre part et surtout la disparition de certains programmes thématiques (voir plus loin). Pour une agence destinée à financer l'ensemble de la recherche nationale, et pas simplement l'excellence comme à l'ERC, le taux de succès est beaucoup trop bas. Pour éviter une sélection aléatoire accompagnée d'une perte de temps et d'une dose de découragement chez les chercheurs, un taux de sélection d'environ $30 \%$ serait bien préférable, moyenne de la DFG en Allemagne.

\section{L'augmentation annoncée des programmes "Blanc " et "Jeunes chercheurs" est fictive.}

En 2010, le budget du programme non thématique a été augmenté à $50 \%$ du budget total de l'ANR. Mais cela a été réalisé par un changement de périmètre, de nombreux projets auparavant présentés dans les programmes thématiques qui ont disparu se retrouvant de fait dans le "Blanc ", avec un taux de succès plus faible qu'auparavant. Les équipes de recherche, pour compenser les aléas, participent à un nombre croissant de projets avec des demandes de financement de plus en plus élevées - jusqu'à $450 \mathrm{k} €$ en moyenne en 2011 et parfois très au-delà - depuis la suppression du maximum imposé à la création de l'ANR. Notons que l'agence, si elle examine les budgets demandés, propose rarement de les réduire et exclut de plafonner les demandes, ce qui pourrait pourtant augmenter les taux de succès.

\section{Les règles imposées ne sont pas adaptées aux vrais besoins des chercheurs.}

La préférence affichée de l'ANR pour les projets collaboratifs oblige souvent à des associations arbitraires, imposant de plus une complexification considérable dans la quête de partenaires quand le besoin réel est celui d'une seule équipe. Un autre défaut est le critère de nouveauté exigé de fait, qui conduit les équipes à proposer toujours des projets sur des sujets nouveaux et rarement la continuation de projets existants, contrairement à ce que fait par exemple la NSF aux États-Unis. Enfin, la durée d'un projet ANR est courte, de 3 ans et au mieux 4 ans, rarement reconduite au-delà, ce qui est souvent insuffisant.

\section{L'évaluation n'est pas assez transparente et pas toujours équitable.}

Enfin, le fonctionnement même des comités d'évaluation de l'ANR fait parfois l'objet de critiques, bien que le recours à des collègues et non à des administratifs soit positif. Certes, plus les thématiques sont larges, plus les interclassements sont difficiles. Cependant, de nombreux biais sont signalés. On peut les attribuer aux effets de mode qui attirent les collègues vers des sujets séduisants, mais aussi au manque éventuel d'impartialité de certains individus dans le comité ou encore aux compétitions territoriales, aggravés par l'anonymat des membres des comités dont la composition n'est connue qu' a posteriori. On signale des cas de fuite des idées, de non-respect de la confidentialité et de conflits d'intérêt non déclarés, directs ou indirects. Enfin, le retour trop bref des expertises auprès des porteurs de projet ne permet pas toujours de justifier suffisamment les décisions prises. Ce dernier défaut pourrait être fortement diminué si le taux de succès était plus élevé et les critères de sélection de ce fait moins arbitraires.

\section{Pour une Agence à la juste place parmi les opérateurs de la recherche}

D'une façon plus générale, on peut s'interroger sur les choix initiaux qui ont présidé à la création de l'ANR. L'Agence vit en circuit fermé, avec ses propres conseils, ses cercles de réflexion stratégiques et une direction autonome. Sa politique est établie sans vraie concertation avec le ministère, les grands organismes ou les universités, ni avec les utilisateurs. Cette tendance s'est trouvée renforcée par la suppression en 2010 de l'USAR, la structure mise en place par le CNRS en soutien à l'ANR pour la gestion d'un certain nombre de programmes, dont le «Blanc ». Or l'évaluation ne peut se passer d'une bonne connaissance des laboratoires et des équipes sur l'ensemble du territoire, qu'il s'agisse de recherche fondamentale, ou orientée vers la compréhension des grands problèmes de l'environnement et de la société, ou encore destinée à des applications innovantes. En Allemagne, par exemple, la DFG travaille en grande concertation avec les scientifiques, tandis que les programmes prioritaires d'importance stratégique, technologique, environnementale ou sociétale sont décidés et gérés directement par le ministère de la recherche et de l'enseignement (BMBF).

Une autre caractéristique de l'ANR est qu'elle est la seule agence de financement généraliste de la recherche en France (avec OSEO pour la recherche industrielle). L'ANR a été chargée d'un ensemble de missions très vaste, en particulier le soutien à des programmes en partenariat avec le privé, dont la qualité scientifique ne paraît pas toujours équivalente à celle des projets non partenariaux et qui favorisent les effets d'aubaine. Il semblerait préférable d'avoir une agence simplement destinée à soutenir la recherche publique qui fonctionnerait comme la DFG, financerait largement des programmes "blancs ", tout en menant une réflexion sur la meilleure façon d'encourager la recherche partenariale et celle dans les entreprises, qui d'ailleurs bénéficient déjà du crédit impôt-recherche.

Nous suggérons qu'au lieu de tout réinventer, une jeune agence de la recherche pourrait s'inspirer des bonnes pratiques des agences étrangères qui ont fait leurs preuves. Placée auprès du ministère de la Recherche, avec des missions resserrées, une plus étroite coordination avec les organismes de recherche et une plus large fraction de programmes "blancs ", elle pourrait envisager des taux de succès satisfaisants. Enfin, rappelons de nouveau que les laboratoires doivent simultanément retrouver des moyens de base adéquats.

Les commentaires sont bienvenus (écrire à leduc@1kb.ens.fr).

Michèle Leduc et Élisabeth Giacobino Laboratoire Kastler Brossel (ENS, UPMC et CNRS), Paris 